\title{
Surface-Based Labeling of Cortical Anatomy Using a Deformable Atlas
}

\author{
Stephanie Sandor and Richard Leahy,* Member, IEEE
}

\begin{abstract}
We describe a computerized method to automatically find and label the cortical surface in three-dimensional (3-D) magnetic resonance (MR) brain images. The approach we take is to model a prelabeled brain atlas as a physical object and give it elastic properties, allowing it to warp itself onto regions in a preprocessed image. Preprocessing consists of boundary-finding and a morphological procedure which automatically extracts the brain and sulci from an MR image and provides a smoothed representation of the brain surface to which the deformable model can rapidly converge. Our deformable models are energyminimizing elastic surfaces that can accurately locate image features. The models are parameterized with 3-D bicubic B-spline surfaces. We design the energy function such that cortical fissure (sulci) points on the model are attracted to fissure points on the image and the remaining model points are attracted to the brain surface. A conjugate gradient method minimizes the energy function, allowing the model to automatically converge to the smoothed brain surface. Finally, labels are propagated from the deformed atlas onto the high-resolution brain surface.
\end{abstract}

Index Terms - Brain atlas, deformable surface models, feature extraction, matching.

\section{INTRODUCTION}

$\mathbf{M}$ $\mathrm{UCH}$ of the anatomical structure of the brain surface is delineated by complex patterns of cortical sulci. These sulcal patterns are routinely used as landmarks for predicting areas of functional localization within the cortex. Since there is not a simple relationship between individual cortical topology and functional localization, activation studies using positron emission tomography (PET), functional magnetic resonance imaging (fMRI), or magnetoencephalography (MEG) must be used to more accurately localize these functional areas. An automated method for extraction of the cortical surface, and subsequent labeling of the major sulci could provide a valuable research tool for the study of the relationship between cortical anatomy and functional localization in individual subjects, and studies of intersubject variability.

This automated procedure could also be used to provide anatomical constraints for use in imaging or localization of

Manuscript received November 10, 1995; revised October 26, 1996. This work was supported by the TRW, Inc. Doctoral Fellowship Program; the National Cancer Institute under Grant RO1 CA59794; the National Institute of Mental Health under Grant RO1 MH53213. An earlier version of this paper was presented at the 1995 International Conference on Information Processing in Medical Imaging. The Associate Editor responsible for coordinating the review of this paper and recommending its publication was J. Duncan. Asterisk indicates corresponding author

S. Sandor is with TRW, Inc., Redondo Beach, CA 90278 USA.

*R. Leahy is with the Signal and Image Processing Institute, Department of Electrical Engineering-Systems, University of Southern California, Los Angeles, CA 90089-2564 USA (e-mail: leahy@sipi.usc.edu).

Publisher Item Identifier S 0278-0062(97)00984-1. functional activity. For example, MEG provides measurements of the magnetic fields produced by neural activity in the brain. These measurements can be used as the basis for imaging or localizing the neural activity which produces these fields. It is generally accepted that the primary current sources which give rise to these evoked fields are confined to cortex and oriented normal to the surface [1]. Hence knowledge from magnetic resonance (MR) images about the location and orientations of cortical folds can be used to improve the MEG current source estimate [2]. Labeling of the cortical surface offers the potential for utilization of probabilistic information about the relationship between cortical anatomy and functional localization.

The goal of our work is to develop a technique that is automatic and accurate in labeling the convoluted regions of the cerebral cortex. In its most basic form, anatomical labeling is performed by an expert tracing out and labeling boundaries of desired structures in an image. This procedure is extremely time consuming and often impractical, particularly for three-dimensional (3-D) data sets. A variety of computerized labeling techniques have been proposed. While some of these techniques require significant user interaction, others have difficulties labeling the more intricate regions of the cerebral cortex.

Many atlas model matching approaches have been proposed for extracting regions from brain images and for anatomically labeling these images. In most existing schemes either rigid models or models with limited ranges of deformation represent a brain atlas and significant user interaction is required to find a correspondence between atlas and image. For example, the stereotactic atlas of Talairach and Tournoux [3] is a widely used manual method of anatomic localization. This atlas is based on an orthogonal proportional grid system centered on the brain's AC-PC line, and atlas dimensions are rescaled using standard landmarks from an individual subject's brain. Rescaling allows a localization of major structures in the brain by assuming a one-to-one correspondence to the spatial location of structures in the scaled atlas. This atlas and procedure can be computerized and would require little user interaction, but a disadvantage of this method is that it is particularly sensitive to intersubject variations in local structure due to the global nature of the scaling procedure.

In the work of Evans et al., structures in an MR image are localized using a template matching procedure [4]. An atlas consisting of standard regions of interest (ROI's), defined on a set of parallel, regularly spaced, planes is globally adjusted to obtain an initial match to a subject's MR image. Individual ROI's on each plane are then scaled, rotated, and translated to 
achieve the best visual fit to the MR image. This procedure, which has been extended to a 3-D volume of interest (VOI) atlas [5], is interactive, since decisions, such as whether or not to translate or scale template regions, must be made by a user.

Since complex brain structures can exhibit significant shape differences between subjects, rigid atlas models cannot accurately account for normal anatomical variation among different brains. The computerized brain atlas described by Greitz et al. [6] employs a similar matching technique to that described in [4], however, this method incorporates into the atlas matching procedure simulated elastic transformations, such as skews and variable scalings. These nonrigid deformations account for large differences in overall brain shape between subjects and improve correspondence between atlas and subject images. As in the work of Evans et al. [4], parameters determining rigid and nonrigid atlas transformations are all chosen interactively.

Bookstein [7] uses a thin-plate spline mechanism to warp an MR image onto a neuroanatomical atlas. An image is treated as labeled point data, i.e., a user identifies certain landmark points in a subject's image, and the image is warped to bring these points into correspondence with the atlas. This technique is employed to study specimen-by-specimen variability around a normative image of the brain.

All these atlas matching techniques are interactive; a user must manipulate the atlas or data to obtain a fit between atlas and subject images; this is a time-consuming operation. An automated technique would significantly decrease the amount of time a specialist must spend with an imaging system. A promising approach for automated matching is to give a model elastic properties and allow it to adapt itself to features of the subject's image. In the work of Bajcsy et al. [8] an atlas is modeled as though it occurs on a rubber sheet and is deformed by forces derived from a feature space representation of a subject's image. This feature space representation emphasizes certain characteristics of an image, usually edges. In this work, subject data consists of X-ray computed tomography (CT) head scans, and automated matching is performed by minimizing a cost functional involving both a deformation constraint and a similarity measure. Extensions of this approach to 3-D data using MR brain images is described by Gee et al. [9]. The deformable volume atlas described by Christensen $e t$ al. [10] is a "textbook" of normal human neuroanatomy which is transformed to fit images of other normal neuroanatomies. These transformations map one volume into another and do not specifically address the problem of matching surface features. In this method, probabilistic transformations on the textbook coordinate system deform the textbook to account for shape differences between the textbook and subject images. The transformations are consistent with the physical properties of deformable elastic solids or viscous fluids. Since the methods in [9] and [10] are based on matching of the raw image volume to an atlas, they can be very sensitive to initialization of the matching procedure and the choice of the parameters governing the deformations. Furthermore, while the viscous-fluid transformations in [10] are guaranteed to preserve topology, they are not guaranteed to map specific features of the atlas, such as cortical sulci, to the corresponding features in the subject anatomy.
All of the matching methods described above are based on matching of the entire brain volume to an atlas. Researchers interested primarily in the shape and organization of the cerebral cortex have concentrated instead on representation of the cortex as a surface. Procedures for unfolding of the cortical surface are described in [11]-[13]. These methods concentrate primarily on providing mappings of the cortical surface and do not directly address the automatic atlas matching problem described here.

The approach we have taken is to model a prelabeled brain atlas as a surface. We parameterize the atlas with 3-D bicubic, B-spline surfaces, and label surface points corresponding to lobe regions and major sulci. This atlas is globally registered (by rotation, translation, and scaling) to a subject's brain and given elastic properties, allowing it to automatically warp itself onto regions in a subject's brain image.

At the heart of our technique, is the assumption that variations in normal brain anatomy from subject to subject can be accounted for by global scaling and local shape differences. Therefore, we label a subject's MR image by matching this image to an anatomic model of a normal brain. From a volume MR image of an anatomically normal brain, we have developed a B-spline surface model of that brain and attached anatomic labels to all points on the surface. To match the atlas model to an image, this spline model is registered to a subject's MR image and treated as a deformable model's initial position. The model is automatically warped to match the subject's image, and labels are transferred from the model to the image.

\section{MR IMAGE PREPROCESSING}

The deformable models which we use in our brain labeling method converge rapidly to unambiguous image features, such as smooth image boundaries. However, matching these models to images containing complex shapes or several neighboring regions is an underconstrained problem, since image contours that are in close proximity to one another can leave the model with many possible local solutions to the boundary-finding task. Thus, in order to constrain the model to warp to the brain surface, we preprocess the MR images.

\section{A. Edge Detection}

In our work, we rely on an edge detector as one step of our preprocessing, because MR images have undesirable characteristics which adversely affect techniques that are based on absolute intensity information. For example, inhomogeneities in the radio-frequency (RF) field generated by an MR scanner can cause voxel intensity values to vary with respect to their location in the image volume. That is, the same tissues can yield different gray-level intensities depending on location. Because of these radiometric variations, tissue classification techniques based on absolute intensity measurements may produce noisy or inaccurate results [14].

Further difficulties in processing MR images come from partial volume effects which blur boundaries and cause apparent connections between anatomically separate regions. Also, problems appear when we attempt to incorporate tissue 
regions' mean intensity values into a region classification procedure. In MR, direct use of region means requires accurate knowledge of appropriate tissue parameters $\left(T_{1}, T_{2}\right.$, and PD) and the specific pulse sequence employed in the image acquisition process. But, it has not yet been shown that MR is a sufficiently quantitative modality to provide accurate in vivo measures of these parameters, notwithstanding that for any given pulse sequence, the ordering of mean tissue intensities e.g., mean (grey matter) $>$ mean (white matter) $>$ mean cerebro-spinal fluid (csf) is usually known.

To detect anatomical boundaries in MR brain images, we decided to resort to a computationally inexpensive preprocessor, the 3-D Marr-Hildreth edge detector [15], [16]. The performance of edge detectors can be problematic, as witnessed by many articles published about the ongoing search for an improved edge detector ([15], [17]-[20]). However, our experience is that the spatial resolution of the Marr-Hildreth filter can be chosen so that the operator is effective in finding a closed brain surface which is a necessary requirement for the input to the morphological procedure described below. We convolve the images with a $7 \times 7 \times 7$ Laplacian of a Gaussian operator with $\sigma=0.75$. Since our images were isotropically resampled to $1-\mathrm{mm}$ cubic voxels, this operator has a spatial dimension of $7 \mathrm{~mm}$ on a side. A binary image is then created by setting zeros crossings to a " 0 " value and all other pixels to " 1 ." The performance of the Marr-Hildreth edge detector can be improved for noisy images by preprocessing the MR volume with a nonlinear anisotropic edge-preserving filter [21], however, this filter was not used for the MR data presented here.

A close examination of the surface extracted using the MarrHildreth operator, reveals that the boundary tends to wander between the true outer brain surface (grey/csf boundary) and the inner cortical surface (grey/white boundary). This is primarily due to the partial volume effects that occur in deep cortical folds in such a way that the two sides of the sulcus appear to merge. While boundary-finding results may be improved using a more sophisticated edge detector, such as the Canny-Deriche method [22], [23], these partial volume effects make it essentially impossible for any edgebased segmentation method to accurately find the outer brain surface without smoothing over the deeper sulci. Since the average thickness of the cortex is on the order of 2-3 mm [24], we anticipate that the boundary mislocation will be tolerable for many applications. Such is the case in which the cortical surface is used to constrain inverse solutions for magnetoencephalographic imaging [2] due to the inherently limited resolution of the modality. If very accurate localization of the outer brain surface is required, this may be achieved either by using a more sophisticated intensity-based segmentation scheme [14], [25] or by iterative relaxation of the brain surface boundary after it is extracted using the morphological methods described below.

\section{B. Morphological Algorithm for Extraction of the Cortex}

Mathematical morphology is an image analysis approach which is directly concerned with measurement of size and shape of objects in an image. Morphological image transformations via set structuring elements are nonlinear filtering operations which locally change the geometry of image objects [26], [27]. When appropriately chosen, morphological operators can simplify an image to a form amenable to feature identification. In our morphological algorithm, we perform operations which: 1) eliminate details in the processed MR head image to develop a smooth template of the overall brain shape and 2) detect sulcal features of the cortical surface.

We use standard notation from mathematical morphology [27], [28] to describe the steps of our algorithm. A dilation of binary set $X$ by structuring element $B$ is denoted $X \oplus B$, and erosion is represented by $X \ominus B$. Morphological opening and closing are denoted by $X \circ B$ and $X \bullet B$, respectively, and we denote a set difference operation between sets $X$ and $Y$ by $X \backslash Y$.

Performing 3-D Marr-Hildreth edge detection on an MR head image results in a binary image, where image pixels corresponding to edges are white ("0") and all others are black ("1"). We represent this binary head image by a 3D set, denoted $X$, comprising the set of black pixels. The brain is then a connected 3-D subset of $X$. To sever unwanted connections and extract only the brain from an image, we first perform an erosion with a 3-D rhombus structuring element of discrete size one $(R 1)$, which is a 3-D digital cross, three voxels wide in the $x, y$, and $z$ directions. Transforming by $R 1$ will eliminate regions which have a size of two voxels or less in any direction and shrink a majority of the brain surface by one voxel. The erosion has the effect of separating brain voxels from surrounding image regions.

Next we perform a 3-D flood filling operation to select only brain voxels. A 3-D flood filling routine finds all voxels connected to a seed point in the brain. Specification of an arbitrary seed point is the only user interaction required for our entire labeling procedure; every other step is automatic. We will denote this morphologically processed and flood filled set of brain voxels as $X_{E \text { Brain }}$.

We fill holes in $X_{E \text { Brain }}$ by first dilating the set with a rhombus structuring element of size one and then closing with an octagon of size two, denoted $O 2$. This isotropic octagon structuring element, which is a digital approximation to a sphere in Euclidean space, has a width of nine voxels. Therefore, these operations will result in a brain volume in which boundaries are smooth and all holes less than nine voxels wide are closed [see Fig. 1(a)]. We will denote the result of this operation $X_{\mathrm{DCBrain}}$

$$
X_{\mathrm{DCBrain}}=\left(X_{E \operatorname{Brain}} \oplus R 1\right) \bullet O 2 \text {. }
$$

Set $X_{\text {DCBrain }}$ is a smooth image that serves as a template on which we can reintroduce fine details from the original binary volume, $X$. That is, when we take the difference $X_{\text {DCBrain }} \backslash X$ we obtain a set which represents all gaps and holes belonging to the brain [see Fig. 1(b)]. We denote this set $X_{\text {Holes. }}$ A difference operation between the closed brain and its set of holes yields our final brain volume

$$
X_{\text {Brain }}=X_{\text {DCBrain }} \backslash X_{\text {Holes }} \text {. }
$$



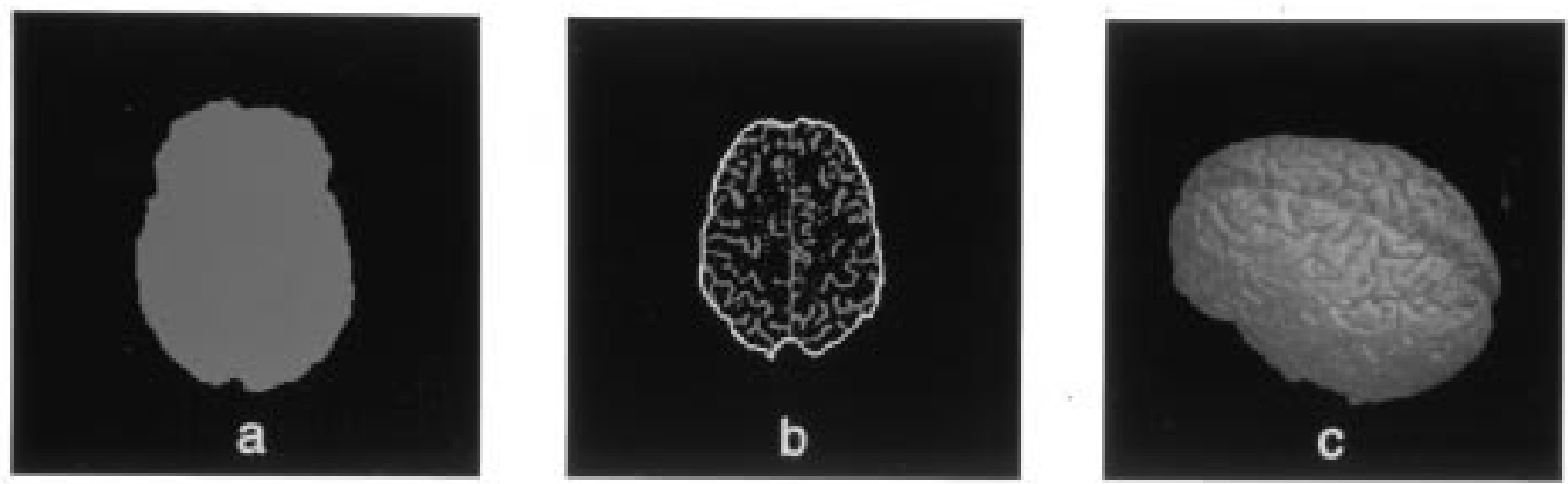

Fig. 1. (a) A transverse slice through the morphologically closed brain surface $\left(X_{\mathrm{DCBrain}}\right)$. (b) A transverse slice showing the detected sulci openings with the boundary of the closed brain surface superimposed on the image $\left(X_{\mathrm{Holes}}\right)$. (c) A surface rendering of a brain which was automatically extracted from MR head images using our morphological algorithm $\left(X_{\text {Brain }}\right)$.

Fig. 1(c) shows a surface rendering of a brain which was automatically extracted using the morphological processing described above.

We summarize five processing steps which we require for extracting a brain from a head image. These steps comprise the first part of our automated algorithm.

1) $X_{\text {Int }}=X \ominus R 1$.

2) Flood-fill $X_{\text {Int }}$ to arrive at $X_{E \text { Brain }}$.

3) $X_{\text {DCBrain }}=\left(X_{E \text { Brain }} \oplus R 1\right) \bullet O 2$.

4) $X_{\text {Holes }}=X_{\text {DCBrain }} \backslash X$.

5) $X_{\text {Brain }}=X_{\text {DCBrain }} \backslash X_{\text {Holes }}$.

The algorithm's next portion labels elements of $X_{\text {Brain }}$ corresponding to cortical regions. The cortical surface is the outer contour of our binary brain image, $X_{\text {Brain }}$, and sulci appear as regions bordering holes and gaps in $X_{\text {Brain }}$. By tracing this outer contour one two-dimensional (2-D) slice at a time, we find portions of $X_{\text {Brain }}$ corresponding to the cortical surface (the outer contour of a binary object is found with a simple boundary following routine). Because the brain has a convoluted surface, finding its outline one slice at a time simplifies cortex-tracing procedures. However, we must take into account the brain's complexity as a 3-D object if we are to process it successfully on a 2-D basis. For example, on certain 2-D slices, deep sulci make the brain appear to be comprised of disconnected regions. Therefore, the outer contour of every patch of connected pixels on every 2-D slice is traced and labeled as part of the cortical surface. We will call the set of surface points found by this procedure $X_{\text {Cortex }}$. Furthermore, because the cortex folds over on itself, on some 2-D slices the cortical surface may appear to be interior to the brain; the contour tracing routine will miss these regions, since it traces only exterior boundaries. Interior regions are found by further morphological processing steps, and the extracted surface is labeled as "sulcus" or "nonsulcus."

In step 4) of our algorithm, we created $X_{\text {Holes }}$, a set representing all gaps and holes in a brain image. A portion of these holes are due to sulci, but others are created by noise or interior brain structures. A feature which differentiates a sulcus opening from any other is that given any point in this opening, we can find a connected path of voxels from that point to the image background which lies outside the brain. If we compare corresponding slices of $X_{\text {Holes }}$ and $X_{\text {DCBrain }}$, we find that sulci openings intersect the boundary of $X_{\text {DCBrain }}$ the closed brain image, somewhere in the volume. We conclude this section by providing a detailed list of necessary steps to find all sulci openings in the cortex. These steps form the second part of our automated algorithm.

1) Trace the boundary of $X_{\text {DCBrain }}$ and initialize the next processing steps on the first slice of $X_{\text {DCBrain. }}$ In addition, initialize a set called $X_{\text {AllSulci }}$ which starts out as an empty set, but after this part of the algorithm is complete, it will contain all voxels corresponding to sulci openings.

2) Find a point on $X_{\text {DCBrain }}$ 's boundary that intersects $X_{\text {Holes }}$ and mark it as a sulcus point.

3) Find all voxels in $X_{\text {Holes }}$ connected to the sulcus point found in step 2) (we denote this set of connected voxels $\left.X_{\text {Sulcus }}\right)$. As in the first part of our algorithm, we use a 3-D flood filling routine to find all voxels connected to a particular point.

4) Perform the following set union: $X_{\text {AllSulci }}=X_{\text {AllSulci }} U$ $X_{\text {Sulcus }}$. That is, add $X_{\text {Sulcus }}$ to the set of all brain sulci.

5) Redefine $X_{\text {Holes }}$ as $X_{\text {Holes }}=X_{\text {Holes }} \backslash X_{\text {Sulcus }}$. That is, remove $X_{\text {Sulcus }}$ from $X_{\text {Holes }}$.

6) Return to step 2), and continue processing in this manner until $X_{\mathrm{DCBrain}}$ volume has been traversed in its entirety.

7) Redefine the set of cortical surface locations as $X_{\text {Cortex }}=X_{\text {Cortex }} \cup X_{\text {AllSulci }}$.

8) Label all surface points on the outer boundary of $X_{\text {AllSulci }}$ as cortical sulci.

Once this processing is complete, we arrive at a set $X_{\text {Cortex }}$ which is a point-by-point description of the cortical surface. Also, $X_{\text {Cortex }}$ is labeled as to which surface points correspond to sulci. Fig. 2(b) shows one slice through $X_{\text {Cortex }}$, the cortical surface found by the above processing steps; Fig. 2(c) shows points that the morphological processing labels as sulci-these points are superimposed on the original gray scale image of the slice.

An advantage of using morphological processing is that we can obtain a smoothed representation of the brain surface. This 

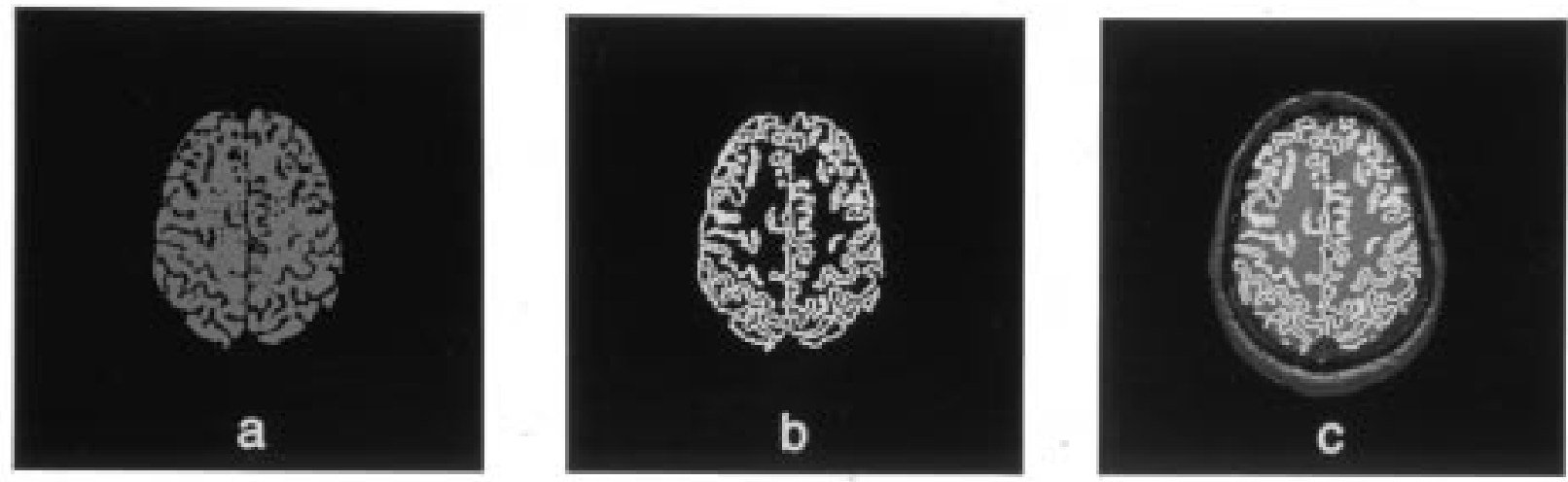

Fig. 2. (a) Transverse slice through extracted binary brain image $\left(X_{\text {Brain }}\right)$ the morphological algorithm $\left(X_{\text {AllSulci }}\right)$.

(b) The extracted cortical surface $\left(X_{\text {Cortex }}\right)$. (c) The sulci detected by

type of representation is necessary because without interactive guidance, deformable models cannot be guaranteed to converge to highly convoluted image features. By performing a morphological closing operation on the extracted brain, we smooth the image, closing all gaps caused by sulci and other cortical structures. Fig. 1(b) shows the boundary of the morphologically closed brain surface superimposed on the sulci image so that we can see where sulci intersect the closed brain surface. Therefore, morphological processing results in a smoothed image to which the model will accurately converge and which still retains information as to where cortical convolutions occur on the brain surface.

\section{THE ATLAS}

The processing steps discussed in Section II extract a pointwise surface description of a brain from an MR image and attach general anatomical (i.e., sulcus or nonsulcus) labels to this surface. In order to perform a more detailed surface labeling, we take advantage of significant a priori knowledge about normal brain anatomy and introduce this knowledge into our processing in the form of an anatomically labeled brain atlas model. Assuming that variations in normal brain anatomy from subject to subject can be accounted for by global scaling and local shape differences, a more detailed labeling is accomplished by matching this atlas model to the morphologically extracted brain surface.

\section{A. Cortical Anatomy}

The convoluted cerebral cortex can be divided into regions or lobes whose boundaries are, for the most part, defined by major cortical fissures; each lobe also has its own specific sulcal patterns. Generally, the external surfaces (i.e., the convex lateral surfaces) of the hemispheres are divided into four lobes: frontal, parietal, occipital, and temporal. Lobe boundaries are formed by anatomical structures, such as the central and lateral sulci, or by artificial lines like the parietotemporal border (see Fig. 3).

Major sulci are critical landmarks since they define the boundaries of lobe regions and serve as rough indicators of functional areas of the cerebral cortex. In normal brains these sulci have the following features: 1) they occur in

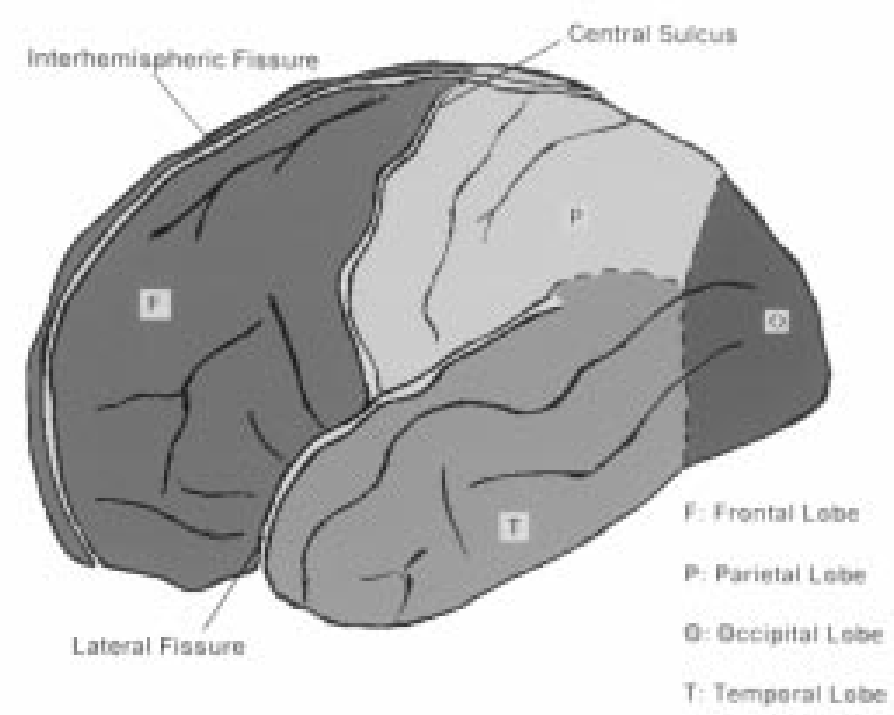

Fig. 3. Major anatomical features on the convex lateral surface of the brain: the four lobes are labeled as $P$-parietal lobe, $F$-frontal lobe, $T$-temporal lobe, and $O$-occipital lobe. The major fissures are indicated, and the boundary between the occipital lobe and its neighboring regions is designated by a dashed line.

approximately the same location and orientation on different subjects; 2) they run long distances across the brain surface without being interrupted by secondary cortical folds; 3) they are the deepest fissures on the cortex. Although they can have quite complex shapes, these sulci are among the most constant features on the brain surface.

Except for major convolutions and fissures, cerebral structures on the surfaces of the hemispheres can be difficult to recognize, due to many secondary folds and interruptions which alter the shape of cortical convolutions. Also, there is a large amount of variability in cortical sulcal patterns, not only among individual brains but also between hemispheres of the same brain; however, basic recurring sulcal patterns can be identified and categorized. For example, the work of Ono et al. gives an in-depth description of sulcal patterns of 25 specimen brains [29]. Certain trends can be found which cause particular sulci to be more readily identified. For instance, part of the reason the central sulcus and Sylvian fissure are 

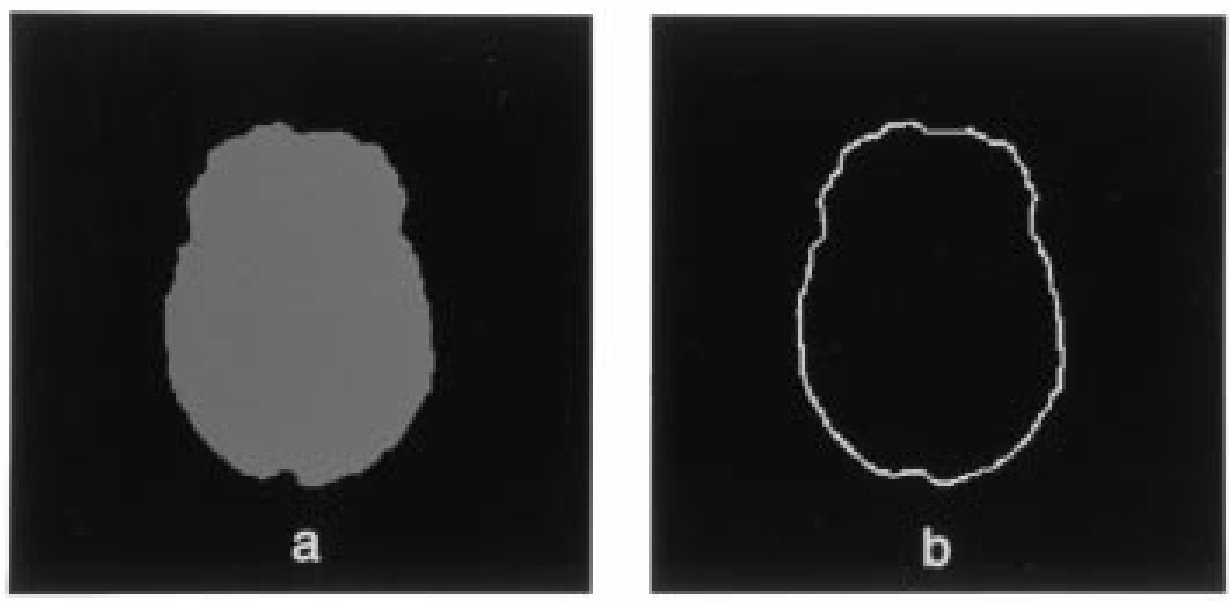

Fig. 4. A contour for one slice of the atlas model. The atlas contours are traced from the outer boundary of a morphologically closed brain.

recognizable is that in the majority of normal brains the sulcus travels from one point to another on the brain surface without being interrupted by a cortical fold. From data gathered on these brain specimens it was found that in $100 \%$ of the cases, the Sylvian fissure was never interrupted and this was true for the central sulcus in $92 \%$ of the specimens.

Many sulci exhibit patterns of frequent interruption, which greatly increase the difficulty of the recognition and labeling task-even for an expert in cortical anatomy. However, additional information about sulci can be used in their identification. The locations of sulci interruption can be important. For example, in the case of the superior frontal sulcus interruptions occur more frequently as one heads toward the extreme end of the frontal lobe. Also, the cerebral fissures have orientations which are typical of lobes in which they lie [3]. That is, the general layout of the cortex is relatively constant among subjects, and once the main sulci (those already included in our atlas) are found, other important sulci can be found since they occur in a particular location and orientation with respect to the major sulci.

\section{B. B-Splines and Atlas Construction}

To develop the atlas model we used a 3-D MR volume image of a male brain which did not show any visible anatomic abnormalities. This image is an updated MRI-version of the original phantom described in [30]. The images were acquired on a 1.5-T GE scanner in a volume acquisition mode. A spoiled grass pulse sequence was used $\left(T_{R}=40 \mathrm{~ms}\right.$ and $T_{E}=13 \mathrm{~ms}$ ) with flow compensation to eliminate artifacts due to moving fluids. The images were isotropically sampled with voxel dimensions of $1.09 \mathrm{~mm}$ in all three directions. The morphological algorithm (see Section II) for extracting a brain from a head image was applied to this MR data.

With morphology we also can find regions of a brain image corresponding to the cortical surface and to sulci openings. By morphologically closing a binary brain image, we obtain a smoothed representation of the brain surface, and it is the outer contour of this smooth surface that we use as our model of the cortex. Fig. 4 shows a slice through the closed brain and the contour which represents the brain atlas on that slice. With results from morphological processing we can find those surface points corresponding to locations of sulci. That is, by taking the intersection of the outer boundary of the closed brain with the set of sulci openings we find points on the closed surface which correspond to locations of different sulci [see Fig. 1(b)]. We then select and label surface points corresponding to the sulci we want included in our atlas, and points lying between these sulci are labeled according to the lobe in which they occur.

By this procedure the atlas surface is described by a list of points representing the outer surface of the brain as it appears on each slice of the closed brain image. Each surface point has an anatomical label attached to it, and we call this collection of points our "original atlas." A B-spline surface representation provides us with a closed form expression for the atlas surface and also requires fewer points than the original atlas description. Hence, we use an approximating B-spline surface to depict our atlas.

A B-spline surface is generated by breaking a surface into a number of patches, representing each patch separately, and joining these patches together to form a continuous surface. The 3-D surface is approximated by summing scaled versions of basis functions, $B_{i, j}(u, v)$, which are piecewise in two parameters [31]. B-spline control vertices, $V_{i, j}=$ $\left(X_{i, j}, Y_{i, j}, Z_{i, j}\right)$, are arranged in a rectangular topology called a control mesh. If $Q(u, v)$ is an approximated surface, then it can be expressed as a double summation

$$
Q(u, v)=(x(u, v), y(u, v), z(u, v))=\sum_{i} \sum_{j} V_{i, j} B_{i, j}(u, v)
$$

In order that $B_{i, j}(u, v)$ have rectangular parametric regions of support, we form basis functions as products of univariate B-splines

$$
B_{i, j}(u, v)=B_{i}(u) B_{j}(v)
$$

These 3-D basis functions are called tensor product B-splines. 


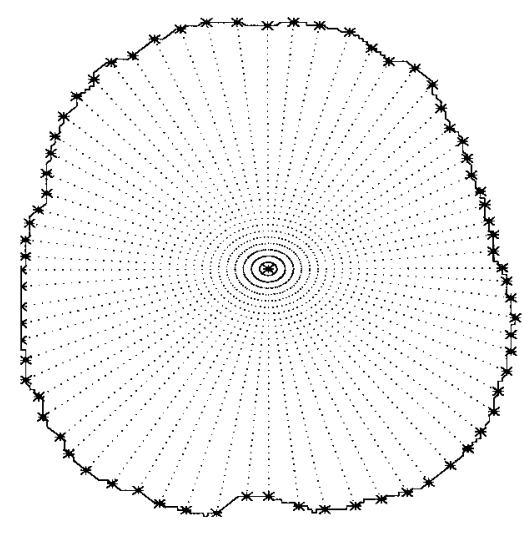

(a)

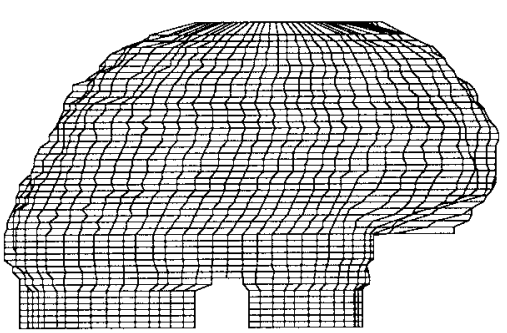

(b)

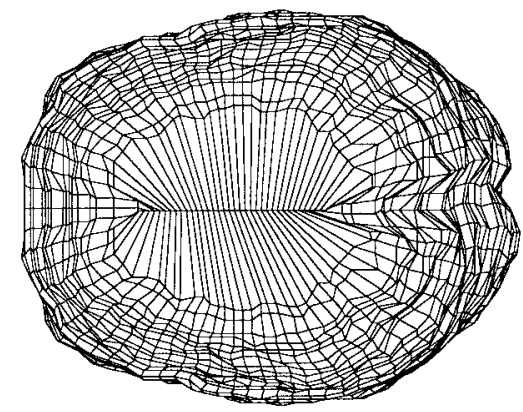

(c)

Fig. 5. (a) Example of the sampling method used to obtain B-spline control mesh. The contour represents one slice of a closed brain image and the asterisks are our sample points which occur at the intersection of the curve with lines drawn from the center of the contour. (b) Lateral view of the atlas' B-spline control mesh obtained with the sampling method shown in (a). (c) Top view of control mesh.

Cubic B-splines, such as the tensor product spline, are well suited for representing nonlinear surfaces. These splines are assembled bicubic surfaces that have positional, first and second derivative continuity ( $C^{2}$ continuity) in every direction at breakpoints. The nonzero portion of a bicubic basis patch spans a region defined by 16 breakpoints in parameter space, allowing local control of a B-spline surface patch, i.e., moving a single control vertex causes a change in only a part of the surface. Furthermore, specification of repeated control vertices or a closed control mesh causes a spline curve to generate predefined behavior at its boundaries.

Our original atlas description consists of lists of points corresponding to closed contours on each slice of a closed brain image. In order to obtain a B-spline control mesh, we sample these contours one slice at a time. Our sampling procedure consists of first taking a closed contour and finding its center. From the center, lines are drawn until they intersect the contour. These intersections are our sample points (see Fig. 5). After the first line, all following lines are drawn from the same center with a $10^{\circ}$ angle separating each line, since we found that this procedure gives a sufficient number of points for our description. These surface sample points serve as the control mesh for a B-spline atlas surface. The control mesh boundaries are formed by tripling boundary control vertices. This tripling of vertices brings the surface closer to the periphery of the control mesh and in fact causes the surface to interpolate the corner vertices of the control mesh. Fig. 5 shows two different $3-D$ views of the atlas control mesh obtained using this sampling technique. We use this method rather than least squares surface spline fitting because this computationally fast and simple technique yields a very regular control mesh whose fidelity to the data can be easily adjusted by increasing or decreasing the number of sample points.

Once we have a control mesh, we evaluate the spline surface $Q(u, v)$ at points in the $u, v$ parameter space, and we obtain a precise description of the surface. Fig. 6 shows two views of the final labeled atlas surface. We attach anatomical labels to all surface points, which in this case numbered close to 5000 points. Specifically, in the atlas presented here we label the lobe points corresponding to the frontal, parietal, and temporal lobes, and sulci points corresponding to the central, lateral, and longitudinal fissures. The occipital lobe was not labeled, due to the difficulty in accurately determining its boundaries, but the lobes and sulci included in the atlas are sufficient to demonstrate our technique of atlas labeling.

\section{DeFORMABle MODElS FOR BRAIN LABELling}

Since brain images are highly complex, contain irregularly shaped regions, and exhibit significant intersubject variation, standard model matching techniques do not perform well on these images. A more promising approach is to use a deformable model, i.e., a brain atlas is modeled as a physical object, given elastic properties, and allowed to warp itself onto a brain surface.

\section{A. 3-D B-Spline Energy Equations for Atlas-Guided Matching}

The deformable models which we incorporate into our system are based on "snakes," active contour models developed by Kass et al. [32]. Snakes are energy-minimizing elastic curves that can accurately locate image features, such as edges. In other words, an energy function is designed whose minima occur at desired image features, and if a snake is placed near a minimum, it falls into it through an energy minimization process. It is important to note that snakes search for local minima instead of a global solution and rely on higher-level processes to place them near a desired solution. In our atlas matching procedure, we define a novel energy function that is designed to ensure that sulci points on the brain model are attracted to sulci points in the subject's image.

We have extended 2-D snakes to 3-D deformable spline models by modifying the snake energy equations and pa- 


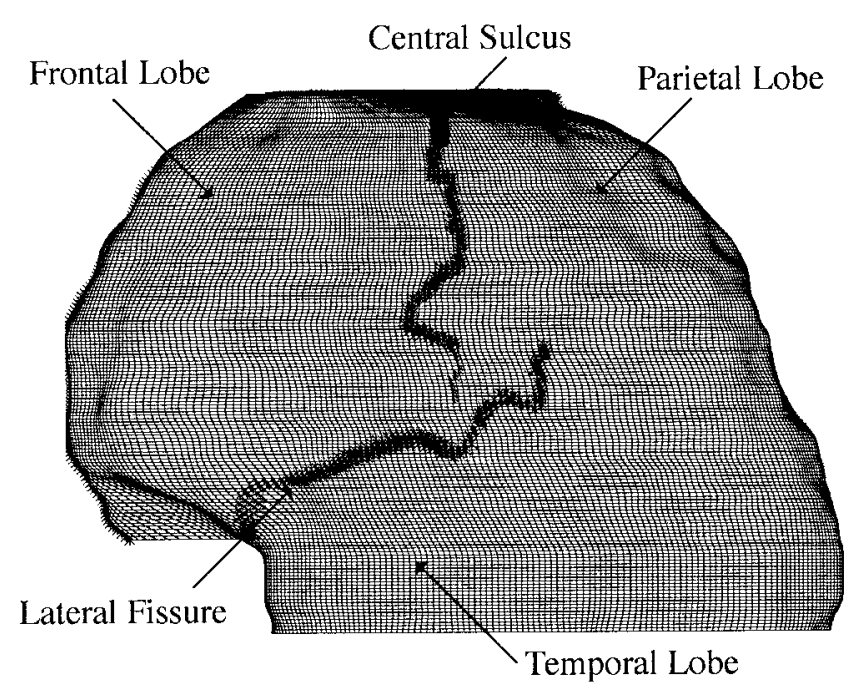

(a)

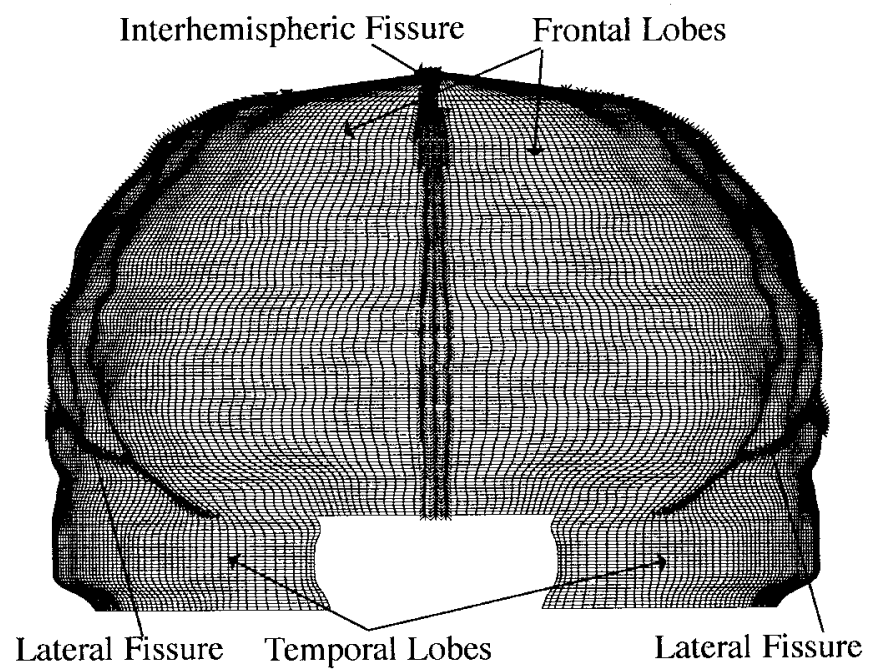

(b)

Fig. 6. A lateral view of the labeled atlas surface. The spline surface has been evaluated, and each surface point has an anatomical label attached to it. The asterisks indicate sulci positions on the surface. (b) A frontal view of the labeled atlas surface.

rameterizing a surface with tensor product B-splines. Let $Q(u, v)=(x(u, v), y(u, v), z(u, v))$ be a parametric description of a surface model, as in (1), and $\bar{Q}(u, v)$ be a parametric description of an atlas model. The total energy can be written

$E_{\text {surface }}(Q)=\int_{0}^{1} \int_{0}^{1}\left(E_{\text {int }}(Q(u, v))+E_{\text {ext }}(Q(u, v))\right) d u d v$.

Internal energy is given by

$$
\begin{aligned}
E_{\text {int }}(Q(u, v))= & \omega_{1,0}\left|\frac{\partial Q}{\partial u}\right|^{2}+\omega_{0,1}\left|\frac{\partial Q}{\partial v}\right|^{2}+2 \omega_{1,1}\left|\frac{\partial^{2} Q}{\partial u \partial v}\right|^{2} \\
& +\omega_{2,0}\left|\frac{\partial^{2} Q}{\partial u^{2}}\right|^{2}+\omega_{0,2}\left|\frac{\partial^{2} Q}{\partial v^{2}}\right|^{2}
\end{aligned}
$$

where coefficients specify mechanical properties of a surface such as elasticity $\left(\omega_{10}, \omega_{01}\right)$, rigidity $\left(\omega_{20}, \omega_{02}\right)$, and twist $\left(\omega_{11}\right)$ [33]. In our application we are interested in matching a brain atlas to individual anatomy. We define the external energy term to reflect the distance between the deformed atlas and the subject's extracted brain surface

$$
E_{\text {ext }}(Q(u, v))=C(Q(u, v))
$$

where $C(Q(u, v))$ is the Chamfer distance (an approximation of the Euclidean distance of each voxel to the nearest edge) between the deformable surface and image features [34]. The discrete form of the total surface energy equation is then

$$
\begin{aligned}
E_{\text {surface }}(Q) & =\sum_{k} \sum_{l}\left[\omega_{10}\left(\sum_{i} \sum_{j} V_{i j} \frac{\partial B_{i j}}{\partial u}\left(u_{k}, v_{l}\right)\right)^{2}\right. \\
& +\omega_{01}\left(\sum_{i} \sum_{j} V_{i j} \frac{\partial B_{i j}}{\partial v}\left(u_{k}, v_{l}\right)\right)^{2} \\
& +2 \omega_{11}\left(\sum_{i} \sum_{j} V_{i j} \frac{\partial B_{i j}}{\partial u \partial v}\left(u_{k}, v_{l}\right)\right)^{2} \\
& +\omega_{20}\left(\sum_{i} \sum_{j} V_{i j} \frac{\partial^{2} B_{i j}}{\partial u^{2}}\left(u_{k}, v_{l}\right)\right)^{2} \\
& +\omega_{02}\left(\sum_{i} \sum_{j} V_{i j} \frac{\partial^{2} B_{i j}}{\partial v^{2}}\left(u_{k}, v_{l}\right)\right)^{2} \\
& \left.+C\left(Q\left(u_{k}, v_{l}\right)\right)\right] .
\end{aligned}
$$

By expressing model energy in terms of a B-spline surface we restrict our search for an energy minimizing surface to a finite dimensional subspace parametrized by the control vertices $V_{i j}$. Note also that the cubic B-spline is the interpolating surface that inherently minimizes the energy corresponding to the integral of the squared second derivatives of a continuous surface. This fact is exploited in the 2-D case by Leitner $e t$ al. [35] to drop the explicit $E_{\text {int }}$ and consider only $E_{\text {ext }}$, using the spline to smooth. However, without the first-order term in the internal energy function the solution to the energy minimization problem may be overly smooth.

For a fully automatic matching procedure, convergence behavior of deformable models must be well understood. In [36], Davatzikos and Prince present a detailed study of convergence and convexity of their own deformable model algorithms. The authors determine model parameters which guarantee both a convex objective function and convergence to the unique global minimum of that function. Convexity is guaranteed only when restrictive conditions are placed on the image data and convergence to a global minimum is dependent upon the starting position of the model. Our energy function, (6), is not convex due to the nonconvexity of the Chamfer distance. In general there will be a number of solutions which represent local minima of $E_{\text {surface }}$. The solution found will depend on the initial placement of the atlas with respect to the 


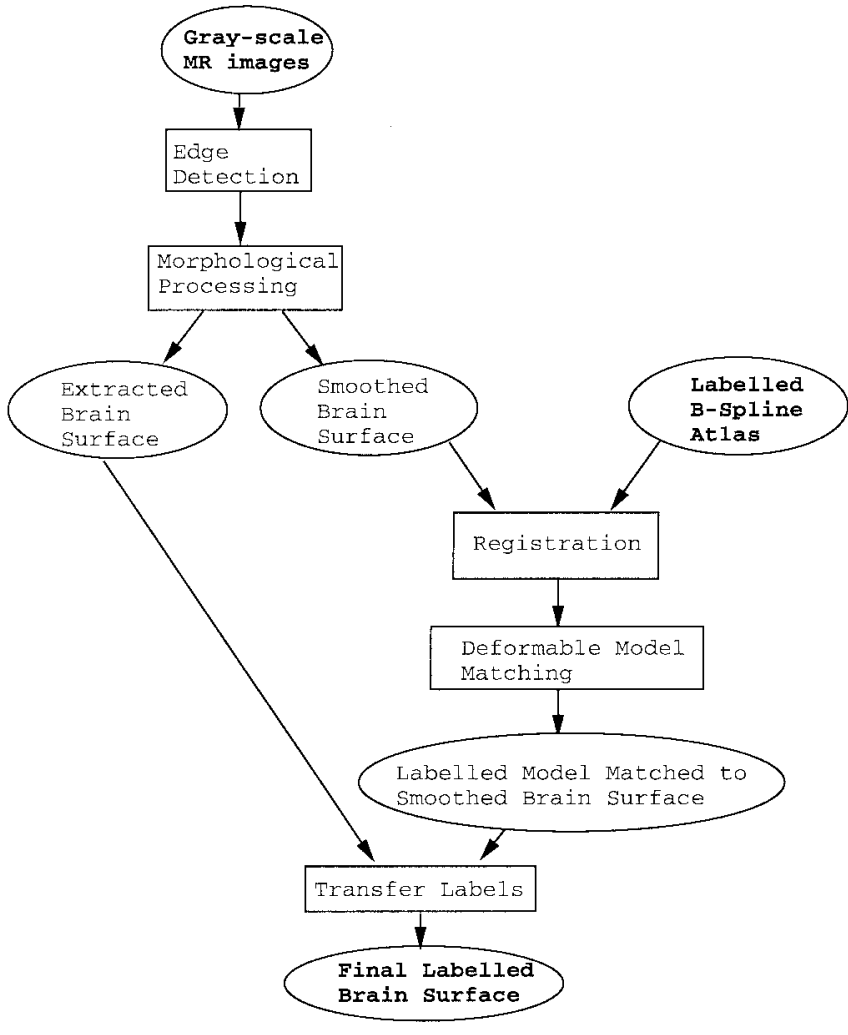

Fig. 7. Flow chart showing an overview of our automatic brain labeling technique.

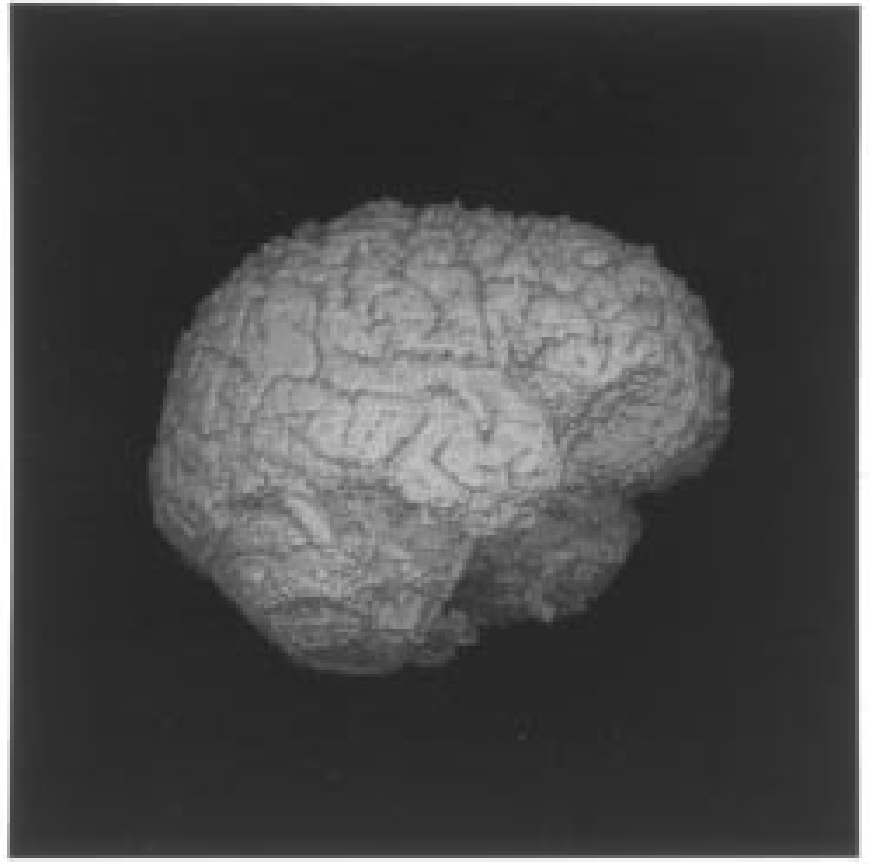

Fig. 8. Surface rendering of the subject's brain. The deformable model is warped to the morphologically closed version of this brain and the anatomical labels are transferred to this high-resolution surface.

extracted surface and the optimization algorithm used (we use a conjugate gradient method). This nonconvexity problem is inherent in the use of active surface models in which distance

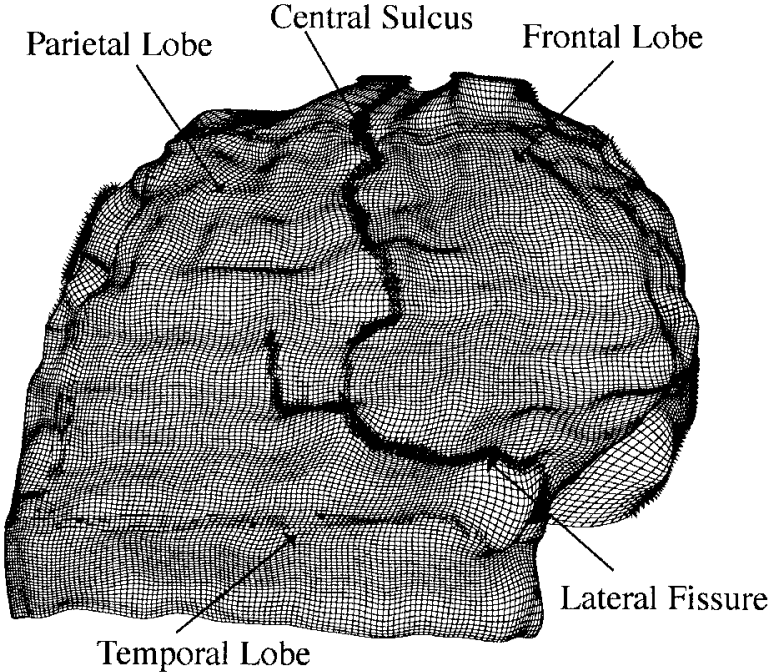

(a)

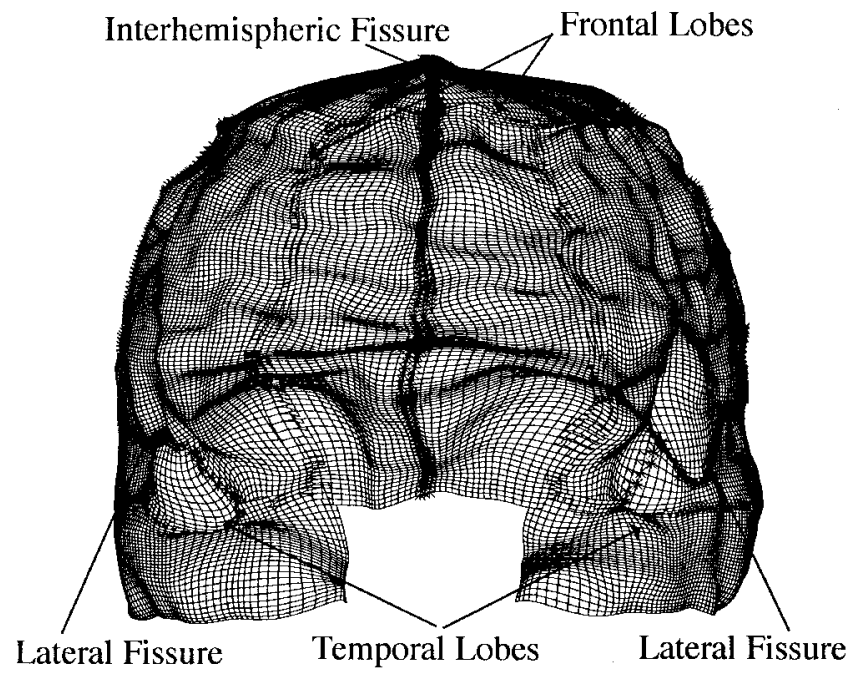

(b)

Fig. 9. (a) Lateral view of the 3-D deformable model after it has been warped to match the subject's brain surface. Each point of the warped surface is anatomically labeled and (b) frontal view of the 3-D deformable model after it has been warped to match the subject's brain surface.

metrics are used to attract the surface to the desired features [33]. However, we note that in contrast to methods that apply active curves and surfaces to grey scale imagery, we are applying the active model to a single surface representing the extracted brain and therefore the atlas will always be attracted to the cortical surface.

\section{MAtChing A 3-D AtLas \\ Model to The Cortical Surface}

The principal goal of this work is to carry out 3-D atlas guided labeling of the cortical surface. In 3-D automatic brain labeling with deformable models, the major problem is to find a method such that the deformable model will converge with high probability to the desired regions. This is the core problem of our work. Without interactive guidance, deformable models can not be assured to converge to highly convoluted brain 

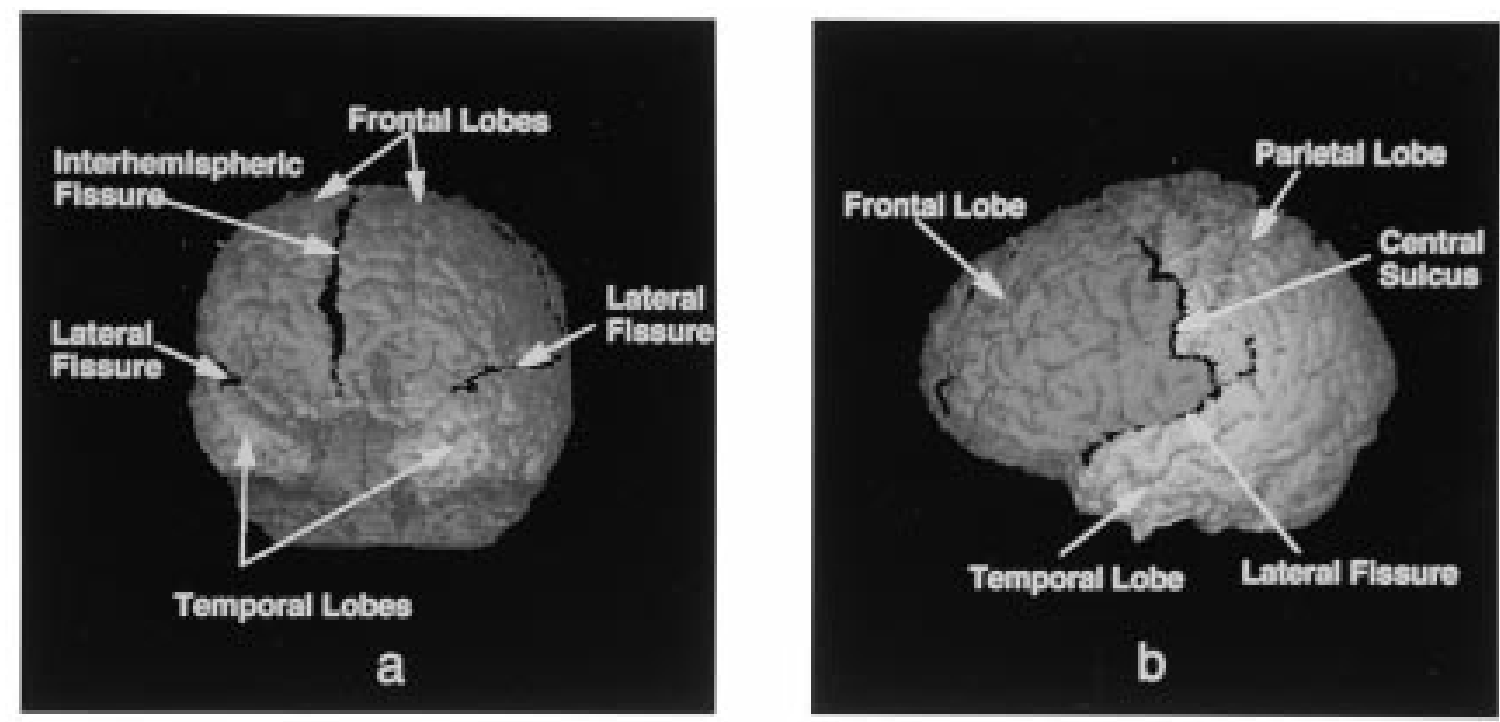

Fig. 10. (a) Frontal view of the final labeled brain surface and (b) lateral view of the final labeled brain surface.

image features. The solution we found is to generate an atlas, which is the initial position of a deformable model, from a smoothed representation of an MR image. We then warp the deformable model to a subject's MR image that has been smoothed with morphological operations equivalent to those used to create the atlas. This avoids the problem of trying to directly match highly convoluted regions in the atlas to highly convoluted regions in the individual MR images. Moreover, we are able to achieve accurate labeling with the smoothed representation of the atlas and subject's brain, using the following procedure: 1) propagating label information from the original atlas brain to its smoothed representation as described in Section III-B; 2) using a novel external energy function that causes sulci in the atlas to warp to sulci in the smoothed individual brains; 3 ) propagating the labels from the warped atlas back to the high-resolution MR brain surface. Fig. 7 shows an overview of this labeling methodology.

To perform 3-D image labeling we use the atlas described in Section III-B (see Fig. 6). This atlas is an anatomically labeled B-spline surface which is registered to a subject's volume MR image. First, the subject's image is resliced along the same planes as the image that was used to create the atlas, then a gross registration is performed by scaling and translating the atlas. This registration consists of creating bounding boxes around both the subject's brain and the atlas brain, where the dimensions of these boxes are determined by the objects' maximum and minimum coordinates in each direction. Linear scaling and translation factors are calculated such that the atlas' bounding box matches the subject brain's bounding box. We then scale and translate the atlas' control mesh by the amount computed from the bounding boxes. These atlas registration operations are performed on the spline control mesh and not on the surface itself, since the surface's shape is invariant with respect to translation, rotation, and scaling of the control mesh. The registered atlas serves as the deformable model's initial position.

In order to warp the B-spline surface, we specify the parameters which control the model's bending. Since the

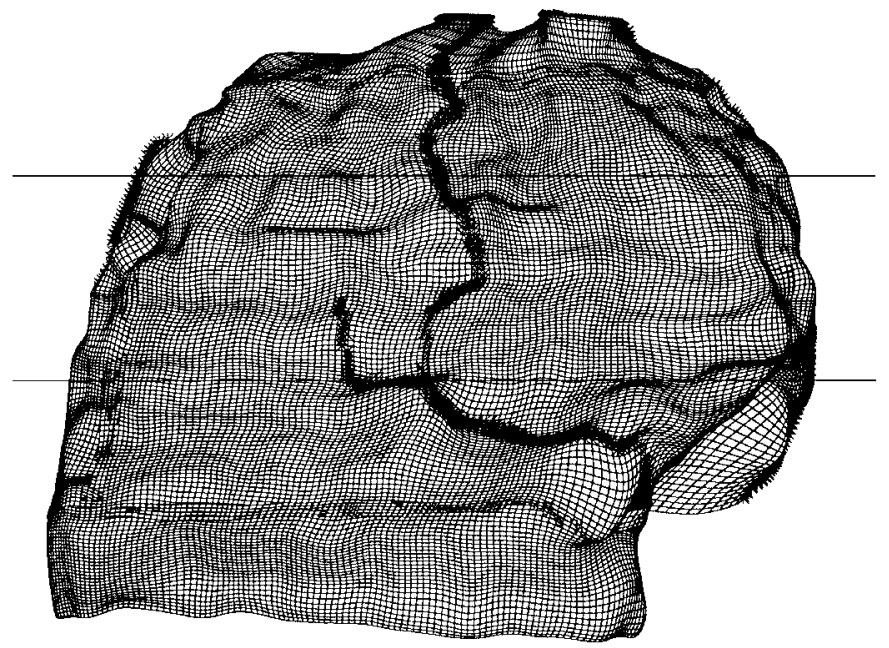

Fig. 11. Lateral view of the warped deformable surface model. The top horizontal line indicates the level at which we have sliced the surface to obtain the images which appear in the following figures.

deformable model is initialized in a shape and location close to its desired final configuration, we weight a model's internal energy terms by parameters computed directly from its initial position. For example, let $\bar{Q}(u, v)$ be a parametric description of an anatomical atlas, which is the initial position of a surface model, then we set model parameters as

$$
\begin{aligned}
& \omega_{1,0}(u, v)=\frac{1}{\left|\frac{\partial \bar{Q}(u, v)}{\partial u}\right|^{2}+\epsilon}, \omega_{0,1}(u, v)=\frac{1}{\left|\frac{\partial \bar{Q}(u, v)}{\partial v}\right|^{2}+\epsilon} \\
& \omega_{1,1}(u, v)=\frac{1}{\left|\frac{\partial^{2} \bar{Q}(u, v)}{\partial u \partial v}\right|^{2}+\epsilon}, \omega_{2,0}(u, v)=\frac{1}{\left|\frac{\partial^{2} \bar{Q}(u, v)}{\partial u^{2}}\right|^{2}+\epsilon} \\
& \omega_{0,2}(u, v)=\frac{1}{\left|\frac{\partial^{2} \bar{Q}(u, v)}{\partial v^{2}}\right|^{2}+\epsilon}
\end{aligned}
$$



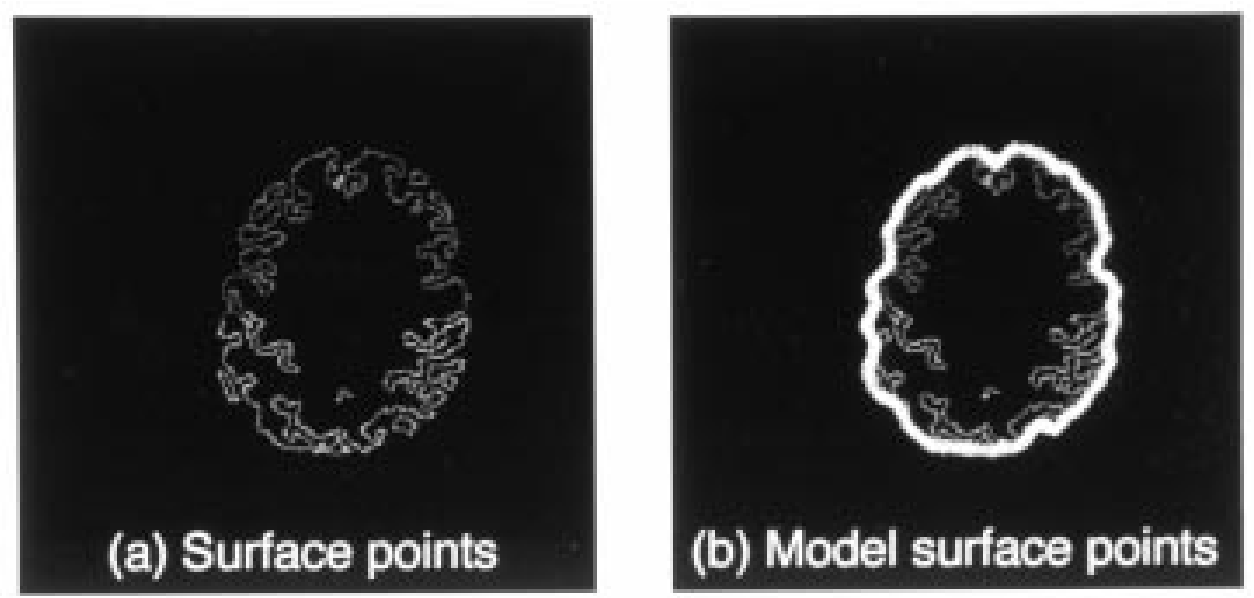

Fig. 12. One 2-D slice through the labeled brain surface. In this example, portions of the frontal lobe, parietal lobe, and major sulci are included in the slice: (a) shows the brain's surface points where different gray levels correspond to different anatomical regions and (b) shows the brain surface with the corresponding slice of the warped atlas model (indicated by asterisks) superimposed on the image. The anatomical labels were transferred from this warped model to the surface points.

where $\epsilon$ is an offset term which prevents a parameter from becoming infinite when a partial derivative goes to zero. We choose $\epsilon$ equal to $0.1 \%$ of the maximum derivative magnitude over the entire surface. This weighting of internal energy terms normalizes a model's bending with respect to an atlas. Thus, if a portion of an atlas is highly twisted, then the corresponding region of a deformable surface can become quite convoluted without incurring a large cost in internal energy. The opposite is true for flat areas of the anatomical atlas, since small movements are subject to a large cost in internal energy. In other words, the model's internal energy weighting parameters are automatically computed from the atlas.

Since the atlas was generated from a smoothed representation of an MR image we warp the deformable model to a subject's smoothed MR image. This type of representation is necessary because without user interaction deformable models can not be guaranteed to converge to complex and convoluted image features. With results from morphological processing we can find those surface points in the subject's MR images which correspond to sulci locations. By finding all openings in the binary brain image and then detecting which openings intersect the outer contour of the closed brain image, we are able to accurately locate sulci on the outer brain surface [see Fig. 1(b)]. Therefore, morphological processing results in a subject's image to which the model will accurately converge and which still retains information as to where cortical convolutions occur on the brain surface.

A Chamfer distance image serves as an external energy function. We use a Chamfer distance function which includes information about sulci locations. We calculate both the Chamfer distance of each pixel to the nearest point on the brain surface $\left(C_{\mathrm{brain}}\right)$ and the Chamfer distance of each pixel to the nearest sulcus point on the outer brain surface $\left(C_{\text {sulcus }}\right)$. If we let $S$ be a set of sulci points on the model, the external energy function can be expressed as

$$
E_{\text {ext }}(Q(u, v))= \begin{cases}C_{\text {sulcus }}(Q(u, v)), & \text { if } Q(u, v) \in S \\ C_{\text {brain }}(Q(u, v)), & \text { otherwise. }\end{cases}
$$

We substitute the external energy term in (7) into the model's discrete energy function (6), and we use the Polak-Ribiere conjugate gradient method for minimizing the model's energy, deforming the model to fit an extracted brain. In this way, model sulci points are attracted to image sulci points, and remaining model points are attracted to the brain surface.

The final step in the labeling procedure is the transfer of anatomical labels from the model to the original (unsmoothed) brain surface. As described in Section III-B, the B-spline surface representing the atlas is evaluated at a number of points in the $u, v$ parameter space, and each of these points has an anatomical label associated with it. Once the model is warped, these same points in parameter space are the locations at which we evaluate the deformable B-spline surface, and thus each point on the deformed model is anatomically labeled. Finally, for each point on the extracted brain surface, we search for the closest point (in Euclidean distance) on the deformed surface and assign its label to the brain surface point.

In Figs. 8-13 we illustrate the application of this atlas matching method to 3-D MR head images. We used the atlas described in Section III-B to label regions on the brain surface corresponding to the frontal, parietal, and occipital lobes and the central, lateral, and interhemispheric sulci (see Fig. 6). The original spline surface was globally registered to match the subject brain's coordinates, where Fig. 8 shows a surface rendering of the subject's morphologically extracted brain. The model was then allowed to deform, and Fig. 9 depicts different views of the warped spline model after it was matched to the morphologically closed brain surface.

Labels were transferred from the deformed model to the original brain surface. Fig. 10 depicts renderings of different views of the final labeled brain surface, where different colors correspond to different anatomical labels. Fig. 11 shows a lateral view of the warped model where the top horizontal line indicates where the slice in Figs. 12 and 13 occurs. Figs. 12 and 13 show that not only the visible outer surface has been labeled but also every other surface point of the 

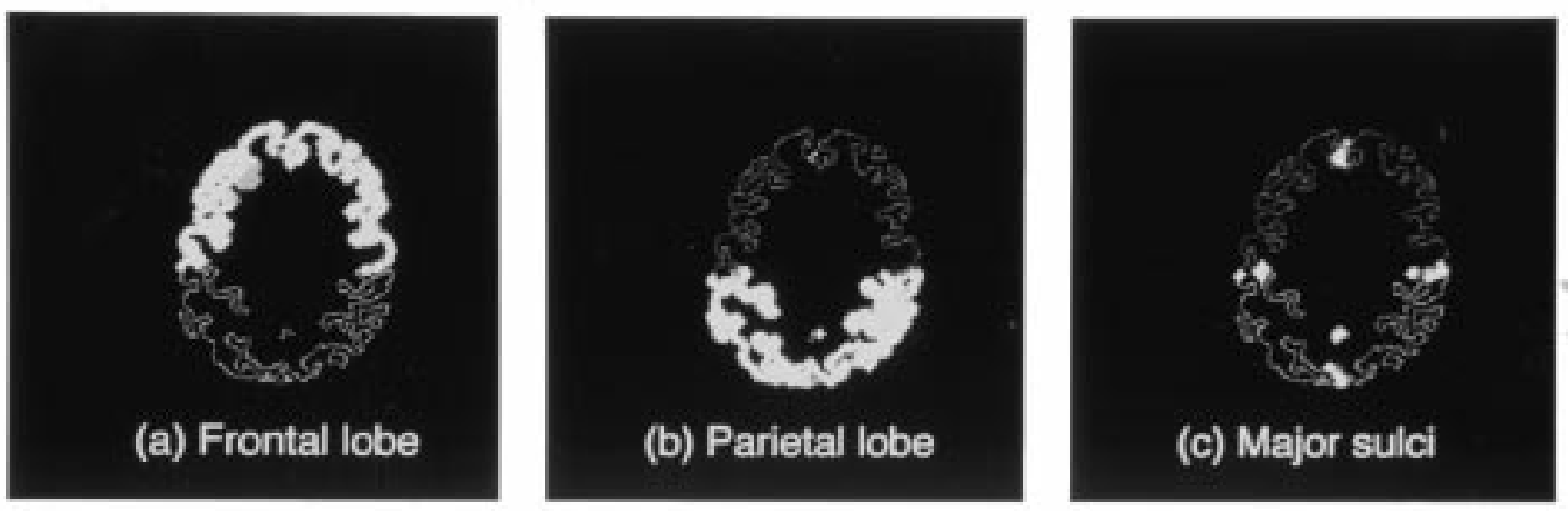

Fig. 13. Labeled surface points. Asterisks highlight the different anatomical labels that were attached to the surface points: (a) shows the points that were labeled as frontal lobe, (b) shows parietal lobe points, and (c) shows points that were labeled as major sulci.
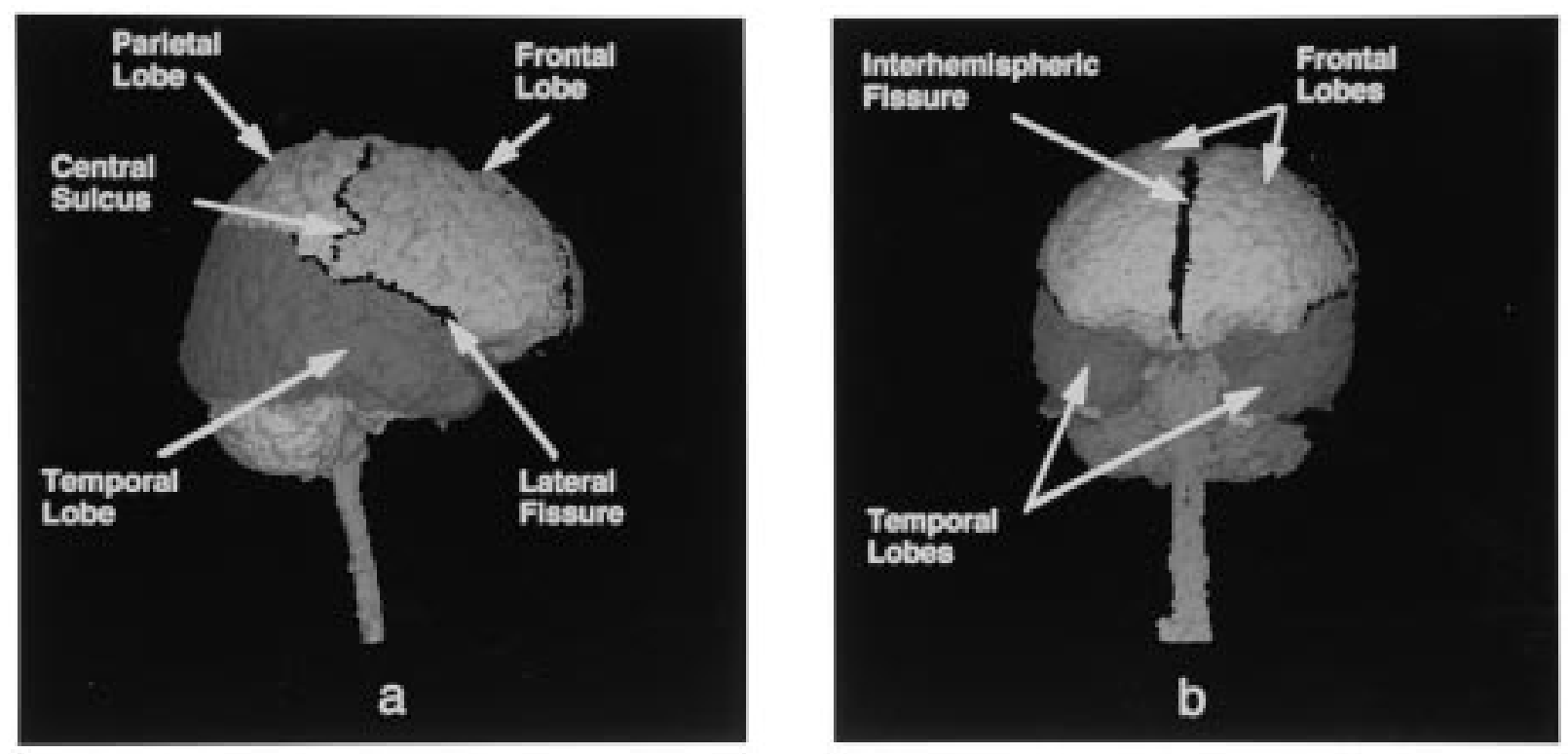

Fig. 14. (a) Lateral view of a final labeled brain surface. The different gray levels correspond to different anatomical labels. (b) Frontal view of the final labeled brain surface.

morphologically extracted brain. Thus, all points which lie on the surfaces of the cortical convolutions are labeled.

In Fig. 14 we show another example of an automatically labeled brain surface. This data was processed using the method described above, except in this case the initial bounding box registration method was replaced with a 12-parameter affine coordinate transformation determined by applying the automated image registration (AIR) software of Woods et $a l$. which is a direct extension to intersubject registration of the method described in [37] for multimodal registration. We found that using this more sophisticated initial registration method lead to some improvement in the labeling, primarily by preventing the temporal lobe label from extending down to the cerebellum.

\section{DISCUSSION AND CONCLUSION}

There are two major problems with using deformable models for 3-D brain labeling. Deformable models cannot be guaranteed to automatically converge to the surface of the brain in raw MR images. In other words, in the presence of neighboring image features, these models may latch on to incorrect boundaries. Also, without user interaction, deformable models can have difficulty converging to complicated object boundaries. Mathematical morphology can help to overcome these problems. Thus, we have developed a morphological algorithm for preprocessing these images. Once boundaries in an MR head image are detected, we apply a specific sequence of morphological operators to extract the brain as a binary object from the head image. Moreover, the morphological processing identifies points which correspond to sulci locations on the brain's surface. By extracting solely the brain we overcome one of the difficulties of matching a deformable model to these images, because we eliminate other anatomical boundaries in the image to which a deformable model may incorrectly converge.

With the images resulting from morphological processing we also overcome the problem of matching a deformable model to a convoluted surface. We generate the atlas, which 
is the initial position of a deformable model, from a smoothed representation of an MR image. Then we warp the deformable model to an extracted brain surface that has been processed with the equivalent smoothing operations as those used in the creation of the atlas. Therefore, the deformable model converges to the smooth outer boundary of the brain's surface. Also, since our morphological processing finds sulci locations on the smoothed brain's surface, we constrain sulci locations on the model to converge to sulci locations on the subject's brain.

Although our approach does not depend on a particular boundary-finding technique, the algorithm is highly dependent on information provided by a low-level processor, and accurate detection of region boundaries is important to all further processing. The Marr-Hildreth operator is accurate in finding brain boundaries that approximate step edges, but like any low-level processor, it is not error free. Portions of the graycsf boundary may be blurred, and the Marr-Hildreth operator does not in general find blurred region boundaries. Also, if misplaced edges cause a sulcus not to satisfy the conditions of our brain model, then that sulcus is not recognized. For these reasons, the brain surface found by morphological operations based on Marr-Hildreth processing may require corrections.

There are improvements and extensions that can be made to our anatomical atlas which will enhance the accuracy of our automatic labeling method. As we indicated in Section III$\mathrm{B}$, our brain atlas was created from MR data acquired from an anatomically normal brain. We labeled this brain by using standard anatomy textbooks to identify brain structures. The results of our automatic labeling procedure depend upon the information in this atlas model; therefore, our model should be checked for correctness by experts in neuroanatomy. Improvements in the accuracy of the atlas model will directly affect the final labeling results. Furthermore, our overall brain extraction and labeling method must be validated by comparing our automatic results with those obtained by an expert. Also, we can measure the sensitivity of our method to low-level processing by comparing the brain surfaces extracted from an image using different boundary-finding techniques.

A modification we can make to our model is to include more anatomical features in the atlas. The atlas we presented in Section III-B contains significant structures on the brain surface; in particular, major sulci are included in this model. Important information can also be obtained by locating and labeling some of the smaller or less-regular sulci. To complete our atlas, the next sulcal features which could be added to it are those large sulci that have a somewhat lower incidence of continuity across the cortex. For example, we could add the inferior frontal, superior frontal, and superior temporal sulci which, respectively, have a $48 \%, 36 \%$, and $32 \%$ uninterrupted rate [29].

The atlas-guided deformable model must be modified to account for these smaller sulci. One way to incorporate additional sulcal patterns into our labeling technique is to extract more information from the subject's morphologically processed brain image before performing deformable atlas matching. Our morphological algorithm labels points on the brain surface which correspond to sulci locations. By tracing connected sulci points, it may be possible to determine whether these points are part of large, uninterrupted sulci and also to find the approximate orientation of the sulci. We could then add further terms to the deformable model's external energy function, thereby allowing model sulci points and image sulci points with similar orientations to be attracted to one another. While we have demonstrated that it is possible to automatically label the major fissures of the brain using a deformable model approach, it is an open question as to whether this approach will also work for those sulci that exhibit a greater degree of intersubject variability.

Finally, the performance of our image labeling technique can be improved by changing our global model to image registration method. Since deformable model behavior is dependent on the model's initial position with respect to image features, a more accurate initial registration technique can improve the model's performance and ensure a correct labeling. For example, we have observed that the 12-parameter affine transformation based on the AIR technique developed by Woods et al. [37] can lead to improvements in the final labeling.

\section{ACKNOWLEDGMENT}

The authors would like to thank G. Zubal of Yale University and C. Aine of Los Alamos Research Lab. for access to volume MR data. They would also like to thank S. Grafton of Emory University for discussions about cortical anatomy and labeling and R. Woods of the University of California, Los Angeles for access to his MR-MR registration software.

\section{REFERENCES}

[1] Y. Okada, "Neurogenesis of evoked magnetic fields," in Biomagnetism: An Interdisciplinary Approach, S. J. Williamson, Ed. New York: Plenum, 1983, pp. 399-408.

[2] A. M. Dale and M. I. Sereno, "Improved localization of cortical activity by combining EEG and MEG with MRI cortical surface reconstruction: A linear approach," J. Cognitive Neurosci., vol. 5, pp. 162-176, 1993.

[3] J. Talairach and P. Tournoux, Co-Planar Stereotaxic Atlas of the Human Brain. Stuttgart, Germany: Georg Thieme Verlag, 1988.

[4] A. C. Evans, C. Beil, S. Marrett, C. J. Thompson, and A. Hakin, "Anatomical-functional correlation using an adjustable MRI-based region of interest atlas with positron emission tomography," J. Cerebral Blood Flow and Metabolism, vol. 8, pp. 513-530, 1988.

[5] A. C. Evans, S. Marrett, J. Torrescorzo, S. Ku, and L. Collins, "MRIPET correlation in three dimensions using a volume-of-interest (voi) atlas," J. Cerebral Blood Flow and Metabolism, vol. 11, pp. A69-A78, 1991.

[6] T. Greitz, C. Bohm, S. Holte, and L. Eriksson, "A computerized brain atlas: Construction, anatomical content, and some applications," J. Comput. Assist. Tomogr., vol. 15, pp. 26-38, Jan./Feb. 1991.

[7] F. L. Bookstein, "Thin-plate splines and the atlas problem for biomedical images," in Proc. 12th Int. Conf. Information Processing in Medical Imaging, July 1991.

[8] R. Bajcsy, R. Lieberson, and M. Reivich, “A computerized system for the elastic matching of deformed radiographic images to idealized atlas images," J. Comput. Assist. Tomogr., vol. 7, pp. 618-625, Aug. 1983.

[9] J. C. Gee, M. Reivich, and R. Bajcsy, "Elastically deformable 3-D atlas to match anatomical brain images," J. Comput. Assist. Tomogr., vol. 17, pp. 225-236, 1993.

[10] G. E. Christensen, R. D. Rabbitt, and M. I. Miller, "3-D brain mapping using a deformable neuroanatomy," Phys. Med. Biol., vol. 39, pp. 609-618, 1994

[11] E. Schwartz, B. Merker, E. Wolfson, and A. Shaw, "Applications of computer graphics and image processing to 2-D and 3-D modeling of the functional architecture of visual cortex," IEEE Comput. Graphics Applicat., pp. 13-23, July 1988. 
[12] C. A. Davatzikos and R. N. Bryan, "Using a deformable surface model to obtain a shape representation of the cortex," in Proc. IEEE Computer Vision Symp., Nov. 1995, pp. 212-217.

[13] G. Carman, H. Drury, and D. V. Essen, "Computational methods for reconstructing and unfolding the cerebral cortex," Cerebral Cortex, vol. 5, pp. 506-517, 1995.

[14] G. Gerig, J. Martin, R. Kikinis, O. Kubler, M. Shenton, and F. Jolesz, "Automating segmentation of dual-echo MR head data," in Proc. 12th Int. Conf. Information Processing in Medical Imaging, July 1991.

[15] D. Marr and E. Hildreth, "Theory of edge detection," Proc. Roy. Soc. London, vol. 207, pp. 187-217, 1980.

[16] M. Bomans, K.-H. Hohne, U. Tiede, and M. Riemer, "3-D segmentation of MR images of the head for 3-D display," IEEE Trans. Med. Imag., vol. MI-9, pp. 177-183, 1990

[17] R. Nevatia and K. R. Babu, "Linear feature extraction and description," Comput. Graphics Image Processing, vol. 13, pp. 257-269, 1980

[18] R. M. Haralick, "Digital step edges from zero crossings of second directional derivatives," IEEE Trans. Pattern Anal. Machine Intell., vol. PAMI-6, pp. 58-68, Jan. 1984.

[19] J. Canny, "A computational approach to edge detection," IEEE Trans. Pattern Anal. Machine Intell., vol. PAMI-8, pp. 679-698, Nov. 1986.

[20] P. Perona and J. Malik, "Scale-space and edge detection using anisotropic diffusion," IEEE Trans. Pattern Anal. Machine Intell., vol. 12, pp. 629-639, July 1990.

[21] G. Gerig, O. Kubler, R. Kikinis, and F. Jolesz, "Nonlinear anisotropic filtering of MRI data," IEEE Trans. Med. Imag., vol. 11, pp. 221-232, June 1992.

[22] R. Deriche, "Using Canny's criteria to derive a recursively implemented optimal edge detector," Int. J. Comput. Vision, pp. 167-187, 1987.

[23] O. Monga, R. Deriche, and J. Rocchisani, "3-D edge detection using recursive filtering: Application to scanner images," Comput. Vision Graphics and Image Processing: Image Understanding, vol. 53, pp. 76-87, Jan. 1991

[24] M. C. Diamond, A. B. Scheibel, and L. M. Elson, The Human Brain Coloring Book. New York: Barnes \& Noble, 1985.
[25] M. X. H. Yan and J. S. Karp, "Segmentation of 3-D brain MR using an adaptive k-means clustering algorithm," in 1994 IEEE Nuclear Science Symp. and Medical Imaging Conference Record, Nov. 1994, vol. 4, pp. 1529-1533.

[26] G. Matheron, Random Sets and Integral Geometry. New York: Wiley, 1975 .

[27] J. Serra, Image Analysis and Mathematical Morphology. New York: Academic, vol. 1, 1982.

[28] R. M. Haralick, S. R. Sternberg, and X. Zhuang, "Image analysis using mathematical morphology," IEEE Trans. Pattern Anal. Machine Intell., vol. PAMI-9, pp. 532-550, Jan. 1987

[29] M. Ono, S. Kubik, and C. D. Abernathey, Atlas of the Cerebral Sulci. Stuttgart, Germany: Georg Thieme Verlag, 1990.

[30] I. G. Zubal, C. R. Harrell, E. O. Smith, Z. Rattner, G. Gindi, and P B. Hoffer, "Computerized 3-dimensional segmented human anatomy," Med. Phys., vol. 21, pp. 299-302, Feb. 1994.

[31] R. H. Bartels, J. C. Beatty, and B. A Barsky, An Introduction to Splines for Use in Computer Graphics and Geometric Modeling. Los Altos, CA: Morgan Kaufmann, 1987.

[32] M. Kass, A. Witkin, and D. Terzopoulos, "Snakes: Active contour models," Int. J. Comput. Vision, vol. 1, pp. 321-331, Jan. 1988.

[33] I. Cohen, L. Cohen, and N. Ayache, "Using deformable surfaces to segment 3-D images and infer differential structures," CVGIP: Image Understanding, vol. 56, pp. 242-263, 1992.

[34] G. Borgefors, "Distance transformations in arbitrary dimensions," Comput. Vision, Graphics, Image Processing, vol. 27, pp. 321-345, 1984.

[35] F. Leitner, I. Marque, S. Lavallee, and P. Cinquin, "Dynamic segmentation: Finding the edge with snake splines," in Curves and Surfaces, P. J. Laurent, A. L. Mehaute, and L. L. Schumaker, Eds. Boston Academic, 1991.

[36] C. A. Davatzikos and J. L. Prince, "An active contour algorithm for mapping the cortex," IEEE Trans. Med. Imag., pp. 65-80, Mar. 1995.

[37] R. P. Woods, S. R. Cherry, and J. C. Mazziotta, "MRI-PET registration with automated algorithm," J. Comput. Assist. Tomogr., vol. 17, pp. 536-546, 1993. 